\title{
Validación del cuestionario RAPID3 en la cohorte argentina ESPAXIA de pacientes con espondiloartritis axial
}

\author{
María Celeste Orozco, Emilce Edith Schneeberger, Natalia Zamora, Fernando A. Sommerfleck, Luis A. Cayetti, \\ Gustavo Citera \\ Sección Reumatología. Instituto de Rehabilitación Psicofísica, Buenos Aires, Argentina.
}

\section{Resumen}

Introducción: El RAPID3 (Routine Assessment of Patient Index Data 3) es un cuestionario sencillo, rápido y de cálculo simple que mostró un buen rendimiento en Artritis Reumatoidea, siendo capaz de reflejar el estado de enfermedad y la calidad de vida en estos pacientes.

Objetivo: Validar el cuestionario RAPID3 en una cohorte de pacientes con EsP axial y evaluar su asociación con otras medidas de evaluación de la enfermedad.

Materiales y métodos: Se incluyeron pacientes consecutivos $\geq 18$ años de edad con diagnóstico de EsPaxial (según criterios NY modificados 1987 y/o ASAS 2009). Todos los pacientes completaron Ios cuestionarios RAPID3, ASQoL, BASDAl y BASFI. La evaluación global de la enfermedad, tanto por el paciente como por el médico, se determinó mediante escala visual análoga (EVA). Se realizó examen físico con recuento articular (44) y evaluación de entesis (MASES). Se obtuvieron muestras de sangre para determinación de HLA-B27 y ERS. Se calculó ASDAS-ERS y SASDAS-ERS. Evaluación radiológica por BASRI por un evaluador ciego al estado clínico de los pacientes $(\mathrm{CCl}=>0,92)$. Se utilizó la versión traducida y validada en Argentina del RAPID3, el cual consiste en 10 preguntas acerca de capacidad funcional y 2 preguntas acerca de dolor y evaluación global de la enfermedad, con sus puntos de corte preestablecidos. Se determinó el tiempo para completar el cuestionario por parte del paciente y el tiempo para calcularlo por parte del médico. Resultados: Se incluyeron 51 pacientes, 39 de sexo masculino (76,5\%), con una edad mediana 42 años (RIC 33-51) y tiempo mediano de evolución de la enfermedad de 20 años (RIC 10,327,6). El 90,5\% presentaba HLA-B27. La mediana del RAPID3 fue 9 (RIC 3-12,8), BASDAI 3,35 (RIC 1,6-6), BASFI 3,4 (RIC 1,15,6), ASQoL 5 (RIC 1-9), SASDAS-ERS 15,9 (RIC 8-22,6), MASES 1 (RIC 0-3) y BASRI 4,5 (RIC 0-11). El cuestionario tuvo excelente reproducibilidad $(\mathrm{CCl}=0,97)$. El tiempo mediano para completar el

\section{Abstract}

Introduction: RAPID3 (Routine Assessment of Patient Index Data 3) is a simple, quick and simple calculation questionnaire that showed good performance in patients with rheumatoid arthritis, being able to reflect the state of disease and quality of life in these patients. Objective: To validate the RAPID3 questionnaire in a cohort of patients with axial spondyloarthritis (axSpa) and assess its association with other measures of the disease.

Materials and methods: We included consecutive patients $\geq 18$ years of age diagnosed with axSpa (according to modified NY criteria 1987 and/or ASAS 2009). All patients completed the RAPID3, ASQoL, BASDAI and BASFI questionnaires. The overall assessment of the disease both by the patient and the doctor was determined by visual analog scale (VAS). Physical examination was performed with joint count (44) and evaluation of enthesis (MASES). Blood samples for determination of HLA-B27 and ERS were obtained. ASDAS-ERS and SASDAS-ERS were calculated. X-rays were evaluated by BASRI by a blinded reader $(\mathrm{CCl}=>0.92)$. The translated and validated in Argentina RAPID3 version was calculated. Time to complete the questionnaire by the patient and time to calculate by the doctor were determined.

Results: 51 patients were included, 39 were male $(76.5 \%)$, median age 42 years (IQR 33-51) and median disease duration of 20 years (IQR 10.3-27.6). 90.5\% had HLA-B27. Median RAPID3 was 9 (IQR 3-12.8), BASDAI 3.35 (IQR 1.6-6), BASFI 3.4 (IQR 1.1-5.6), ASQoL 5 (IQR 1-9), SASDAS-ESR 15.9 (IQR 8-22.6), MASES 1 (IQR $0-3)$ and BASRI 4.5 (IQR 0-11). The questionnaire had excellent reproducibility ( $\mathrm{ICC}=0.97$ ). The median time to complete the RAPID3 was 2 minutes (IQR 0.91 to 3), and to calculate 10 seconds (IQR 6-15). RAPID3 had very good correlation with SASDAS ESR $(r: 0.87)$, BASDAI ( $r: 0.89)$, BASFI $(r=0.8)$ and ASQoL $(r=0.83)$ and good with MASES ( $r: 0.58)$. In multiple linear regression, using total RAPID score as dependent variable and adjusting for age, sex and 
RAPID3 fue de 2 minutos (RIC 0,91-3), y para calcularlo de 10 segundos (RIC 6-15). Se observó muy buena correlación del RAPID3 con SASDAS ERS ( $r: 0,87)$, BASDAI (r:0,89), BASFI (r:0,8) y ASQoL $(r: 0,83)$ y buena con el MASES ( $r: 0,58)$. Al evaluar los puntos de corte preestablecidos del RAPID3 y el SASDAS ERS, observamos buena concordancia entre los mismos (Kappa:0,5, $p=0,0001$ ). También encontramos muy buena asociación de los puntos de corte del RAPID3 y el BASDAI $(p=0,0001)$. En la regresión lineal múltiple, utilizando como variable dependiente el puntaje total RAPID-3, ajustando por edad, sexo y tiempo de evolución de la enfermedad, se observó una asociación significativa con BASDAI (coef $\beta$ : 0,55, $p=0,0001$ ), BASFI (coef $\beta 0,25, p=0,008$ ), ASQoL (coef $\beta: 0,22$, $p=0,02$ ), como también con SASDAS ERS (coef $\beta: 0,42, p=0,001$ ). Conclusión: El RAPID3 es un cuestionario válido, confiable y reproducible para ser utilizado en EsP axial, simple para completar y calcular. Y además, tiene la ventaja de reflejar el estado de tres aspectos importantes de la enfermedad: actividad, capacidad funcional y calidad de vida.

Palabras clave: RAPID3, ESPAXIA, Argentina, espondiloartritis axial. disease duration, a significantassociation was observed with BASFI $(\beta$ coeff $0.25, p=0.008$ ), ASQoL ( $\beta$ coeff: $0.22, p=0.02$ ), and mainly with SASDAS ERS ( $\beta$ coeff: $0.42, p=0.001)$ and BASDAI $(0.55, p=0.0001)$. Conclusion: RAPID3 is a valid, reliable and reproducible questionnaire to be used in axSpa. It is simple to complete by the patient and to evaluate by the rheumatologist.

Key words: RAPID3, ESPAXIA, Argentina, axial spondyloarthritis.

\section{Introducción}

Las Espondiloartritis (EsP) son enfermedades inflamatorias, que comparten diversas características como compromiso axial, artritis periférica, entesitis, dactilitis, presencia de HLA-B27 y ausencia de Factor Reumatoideo (FR). Dentro de este grupo de enfermedades se incluyen la Espondilitis Anquilosante (EA), Artritis Psoriásica (APs), la Artritis Reactiva (ARe), la Espondiloartritis asociada a enfermedad inflamatoria intestinal (EII) y la Espondiloartritis Juvenil (EsPj).

En la Espondiloartritis axial (EsPax), la actividad persistente de la enfermedad se asocia a una significativa disminución de la capacidad funcional con el consecuente deterioro de la calidad de vida. Tanto la observación sistemática como la implementación de instrumentos que permitan obtener información cuantitativa son de suma importancia, dado que proveen información más exacta sobre la evolución y pronóstico de los pacientes.
Existen diferentes autocuestionarios que fueron diseñados para evaluar los distintos aspectos de la enfermedad como el BASDAI $^{1}$ para la actividad de la enfermedad, BASFI $^{2}$ para valoración de la capacidad funcional, y ASQoL ${ }^{3}$ para calidad de vida.

En el año 2008, Pincus T. y cols. ${ }^{4}$ desarrollaron el autocuestionario RAPID3 (Routine Assessment of Patient Index Data 3) que consiste en 10 preguntas acerca de capacidad funcional y 2 preguntas respecto a dolor y evaluación global de la enfermedad. Este es un autocuestionario sencillo, rápido y de cálculo simple que mostró un buen rendimiento en Artritis Reumatoidea, siendo capaz de reflejar el estado de la enfermedad y la calidad de vida en estos pacientes ${ }^{4-7}$.

Si bien el RAPID3 inicialmente fue utilizado en Artritis Reumatoidea (AR), también su rendimiento fue probado en pacientes con otras enfermedades reumáticas como Osteoartritis (OA), Lupus Eritematoso Sistémico (LES), Espondiloartritis (EsP) y Gota ${ }^{8}$. 
El objetivo de nuestro estudio fue validar el cuestionario RAPID3 en una cohorte de pacientes con EsPax y evaluar su asociación con diferentes medidas de evaluación de la enfermedad.

\section{Materiales y métodos}

Se incluyeron pacientes consecutivos $\geq 18$ años de edad con diagnóstico de EsPax (según criterios NY modificados 1987 y/ASAS 2009) de la cohorte argentina ESPAXIA (Estudio de eSPondiloartritis Axial IREP Argentina). Cada paciente aceptó su participación en el estudio mediante la firma de consentimiento informado y el estudio fue aprobado por el comité de ética del hospital. Se consignaron datos sociodemográficos (edad, sexo, estado civil, ocupación, años de educación), datos relacionados con la enfermedad (tiempo de evolución de la enfermedad, manifestaciones extraespinales, comorbilidades, tratamiento).

A los pacientes les fueron administrados los siguientes cuestionarios: BASDAI ${ }^{1}$ (Bath Ankylosing Spondylitis Disease Activity Index), BASFI ${ }^{2}$ (Bath Ankylosing Spondylitis Functional Index) ASQoL ${ }^{3}$ (Ankylosing Spondylitis Quality of Life), y la versión traducida al español y validada en Argentina del RAPID39. Los cuestionarios BASFI, BASDAI, y ASQoL fueron previamente traducidos y validados en nuestro centro ${ }^{10,11}$. Los puntos de corte establecidos del RAPID3 son: $>12$ : alta actividad; 6,1-12: moderada actividad; 3,1-6: baja actividad y <3: remisión. Además, se determinó el tiempo para completar este cuestionario por parte del paciente y el tiempo para calcularlo por parte del médico. La evaluación global de la enfermedad tanto por el paciente como por el médico se determinó mediante escala visual numérica (EVN). Se realizó examen físico con recuento articular de 44 articulaciones. El compromiso entésico se evaluó mediante índice de $\mathrm{MASES}^{12}$. Se consignaron además los reactantes de fase aguda Eritrosedimentación (ERS) y proteína $\mathrm{C}$ reactiva $(\mathrm{PCR})$ correspondientes a la fecha de la visita. Se consignó la presencia de HLA-B27.

Se realizó el cálculo del índice compuesto ASDAS-ERS (Ankylosing Spondylitis Disease Activity Score) $)^{13-15}$ y SASDAS-ERS (Simplified Ankylosing Spondylitis Disease Activity Score $)^{16,17}$.

Se clasificó a los pacientes según los estados de actividad de la enfermedad mediante ASDAS y SASDAS en: muy alta, alta, moderada actividad y enfermedad inactiva. La evaluación radiológica fue realizada mediante BASRI ${ }^{18}$, por un evaluador ciego al estado clínico de los pacientes, que mostró previamente un coeficiente de correlación (CCI) de 0,92.

Para el análisis estadístico, las variables continuas se expresaron como medianas $(m)$ con su correspondiente rango intercuartilo (RIC) o como medias y desvío standard (DS), y las variables categóricas en porcentaje y frecuencia. Las variables categóricas se compararon mediante test de $\mathrm{Chi}^{2}$ y test exacto de Fisher, y las variables continuas por test de Mann-Whitney y Kruskal Wallis. Correlaciones por test de Spearman. A 10 pacientes se les efectuó un segundo RAPID3 a los 3 días del primero y la reproducibilidad del cuestionario se evaluó mediante Coeficiente de Correlación Intraclase (CCI). Finalmente, se realizó una regresión lineal múltiple utilizando como variable dependiente el RAPID3 para evaluar la asociación del mismo con los diferentes aspectos de la enfermedad. Un valor de $\mathrm{p}<0,05$ fue considerado significativo en todos los casos. Se utilizó el software SPSS versión 21.

\section{Resultados}

Se incluyeron 51 pacientes, de los cuales 39 eran de sexo masculino (76,5\%), con una edad mediana de 42 años (RIC 33-51) y un tiempo mediano de evolución de la enfermedad de 20 años (RIC 10,3-27,6). El 90,5\% presentaba HLA-B27 positivo. La escolaridad mediana fue de 13 años (RIC 10-17).

Treinta y seis pacientes $(70,6 \%)$ tenían EA pura, 7 $(13,7 \%)$ EsP asociada a Psoriasis, 4 (7,8 \%) EsPj y en 2 pacientes (3,9\%) ARe y EsP asociada a EII, respectivamente.

En cuanto al tratamiento, 40 pacientes $(81,6 \%)$ se encontraban en tratamiento con AINEs, 8 (16,3\%) con Drogas Modificadoras de la Enfermedad (DME) y 21 $(43,8 \%)$ con agentes anti-TNF $\alpha$.

Las características clínicas y sociodemográficas se describen en la Tabla 1.

El RAPID3 tuvo excelente reproducibilidad (CCI=0,97). El tiempo mediano para completar el RAPID3 fue de 2 minutos (RIC 0,91-3), y el tiempo mediano de cálculo fue de 10 segundos (RIC 6-15). El RAPID3 tuvo muy buena correlación con las medidas de los diferentes aspectos de la enfermedad: SASDAS ERS (Rho:0,87), BASDAI (Rho:0,89), BASFI (Rho:0,8) y ASQoL (Rho:0,83) y buena con el índice de entesitis MASES (Rho:0,58) (Tabla 2).

Además, se observó una asociación significativa de los 


\begin{tabular}{|l|c|}
\hline VARIABLES & $\mathrm{N}=51$ \\
\hline Sexo masculino & $39(76,5)$ \\
\hline Edad años $\boldsymbol{m}$ (RIC) & $42(33-51)$ \\
\hline Tiempo evolución años (RIC) & $20(10,3-27,6)$ \\
\hline Escolaridad años $\boldsymbol{m}$ (RIC) & $13(10-17)$ \\
\hline HLA B27 positivo $\boldsymbol{n}$ (\%) & $46(90,2)$ \\
\hline ERS $\boldsymbol{m} \boldsymbol{m} / \mathbf{h} \boldsymbol{m}$ (RIC) & $11(6-21,5)$ \\
\hline RAPID3 $\boldsymbol{m}$ (RIC) & $\mathbf{9 ( 3 - 1 2 , 8 )}$ \\
\hline BASDAI $\boldsymbol{m}$ (RIC) & $3,35(1,6-6)$ \\
\hline BASFI $\boldsymbol{m}$ (RIC) & $3,4(1,1-5,6)$ \\
\hline ASQoL $\boldsymbol{m}$ (RIC) & $5(1-9)$ \\
\hline SASDAS- ERS $\boldsymbol{m}$ (RIC) & $15,9(8-22,6)$ \\
\hline MASES $\boldsymbol{m}$ (RIC) & $1(0-3)$ \\
\hline BASRI $\boldsymbol{m}$ (RIC) & $4,5(0-11)$ \\
\hline$m$ (mediana) & \\
\hline
\end{tabular}

\begin{tabular}{|l|c|c|c|c|c|c|}
\hline & RAPID3 & SASDAS-ERS & BASDAI & BASFI & ASQoL & MASES \\
\hline $\begin{array}{l}\text { RAPID3 } \\
\text { Rho } \\
\text { Spearman }\end{array}$ & 1 & 0,869 & 0,896 & 0,813 & 0,854 & 0,589 \\
\hline $\begin{array}{l}\text { SASDAS-ERS } \\
\text { Rho } \\
\text { Spearman }\end{array}$ & 0,869 & 1 & 0,923 & 0,702 & 0,749 & 0,506 \\
\hline $\begin{array}{l}\text { BASDAI } \\
\text { Rho } \\
\text { Spearman }\end{array}$ & 0,896 & 0,923 & 1 & 0,716 & 0,803 & 0,564 \\
\hline $\begin{array}{l}\text { BASFI } \\
\text { Rho } \\
\text { Spearman }\end{array}$ & 0,813 & 0,702 & 0,716 & 1 & 0,771 & 0,438 \\
\hline $\begin{array}{l}\text { ASQ0L } \\
\text { Rho } \\
\text { Spearman }\end{array}$ & 0,834 & 0,749 & 0,803 & 0,771 & 1 & 0,545 \\
\hline $\begin{array}{l}\text { MASES } \\
\text { Rho } \\
\text { Spearman }\end{array}$ & 0,589 & 0,506 & 0,564 & 0,438 & 0,545 & 1 \\
\hline
\end{tabular}

Tabla 1. Características sociodemográficas y clínicas de los pacientes.

Tabla 2. Correlación RAPID-3 con otras medidas de evaluación.

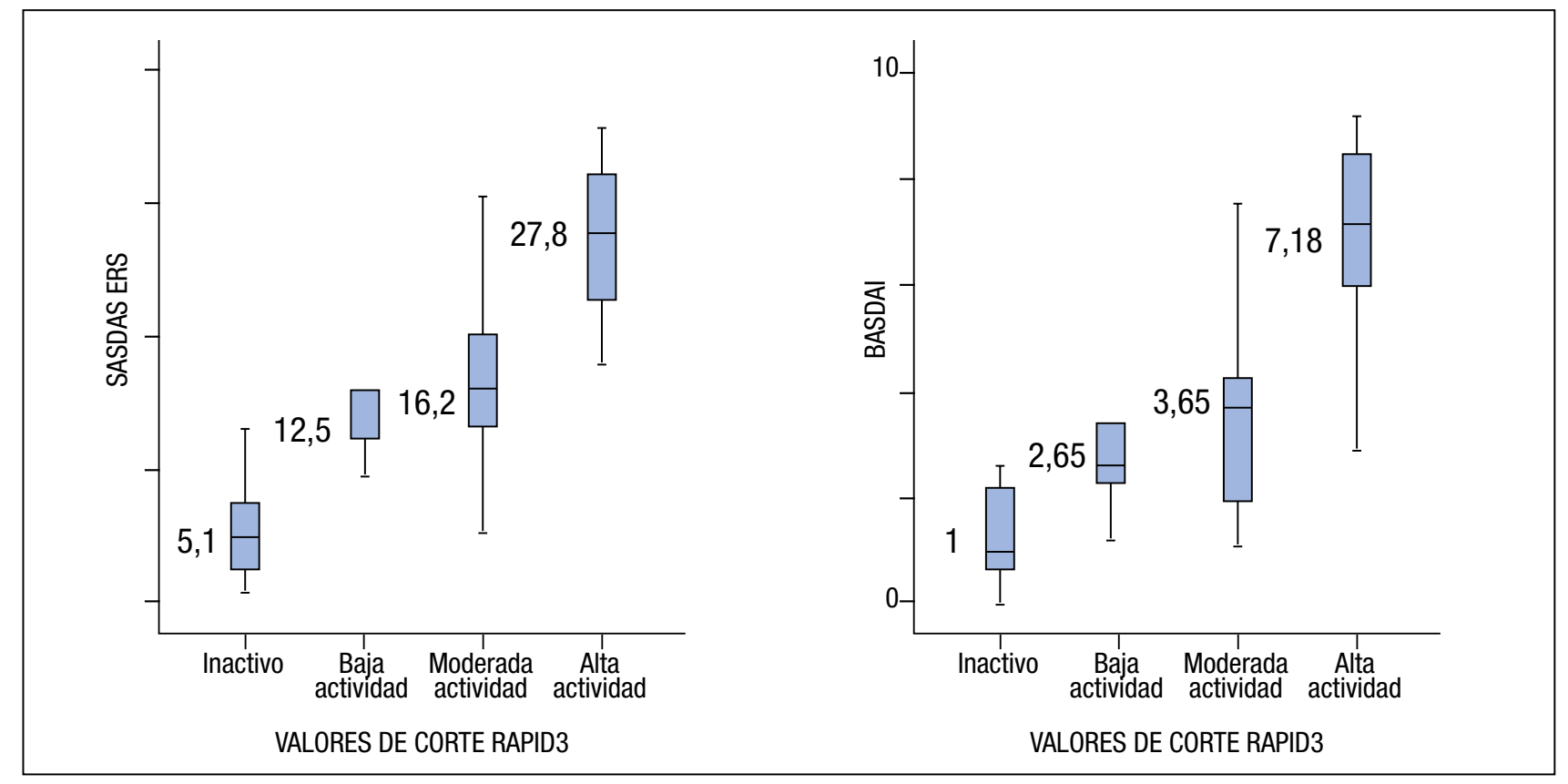

Figura 1. Distribución del SASDAS-ERS y el BASDAl según los puntos de corte del RAPID3.

diferentes puntos de corte del RAPID3 con SASDAS-ERS y BASDAI (Figura 1).

En la regresión lineal múltiple, utilizando el puntaje total RAPID3, como variable dependiente, ajustando por edad, sexo y tiempo de evolución de la enfermedad, se observó una asociación significativa con BASDAI (coef $\beta: 0,55, p=0,0001$ ), BASFI (coef $\beta: 0,25, p=0,008)$, ASQoL (coef $\beta: 0,22, p=0,02$ ), así como también con SASDAS ERS $(\operatorname{coef} \beta: 0,42, \mathrm{p}=0,001)$ (Tabla 3).

\begin{tabular}{|l|c|c|c|}
\hline VARIABLES & $\boldsymbol{\beta}$ & Error típico & $\mathbf{P}$ \\
\hline Edad & $-0,22$ & 0,031 & 0,701 \\
\hline Tiempo evolución & 0,017 & 0,039 & 0,779 \\
\hline Sexo & $-0,007$ & 0,866 & 0,895 \\
\hline BASDAI & 0,548 & 0,240 & 0,0001 \\
\hline BASFI & 0,252 & 0,220 & 0,008 \\
\hline ASQoL & 0,224 & 0,138 & 0,027 \\
\hline
\end{tabular}

Variable dependiente: RAPID3.

Tabla 3. Regresión lineal múltiple. Variables asociadas a RAPID3. 


\section{Discusión}

En el presente estudio, demostramos que el cuestionario RAPID3, sibienfue desarrollado inicialmente para pacientes con AR, es un índice válido, confiable y reproducible para ser utilizado en pacientes con diagnóstico de EsPax.

Hasta el momento, en los pacientes con EsPax, la evaluación de los diferentes aspectos de la enfermedad no era posible con la realización de un solo cuestionario. Y el resultado del RAPID3 refleja el estado de tres aspectos importantes de la enfermedad a considerar como: la actividad de la enfermedad, la capacidad funcional y la calidad de vida. Este hallazgo coincide con nuestro estudio de validación del cuestionario en pacientes con $\mathrm{AR}^{9}$.

Nuestro trabajo demostró una muy buena correlación del RAPID3 con otras medidas de actividad de la enfermedad, tales como SASDAS ERS, BASDAI, confirmando resultados de estudios previos, en los cuales concluyen que el RAPID3 es tan útil como BASDAI, ASDAS en pacientes con EA, sugiriendo su utilización como parte de la rutina en la atención médica ${ }^{19-21}$.

Interesantemente, observamos muy buena asociación de los puntos de corte de RAPID3 con el SASDAS-ERS y BASDAI, confirmando la validez de sus puntos de corte.

Otra cualidad importante de este cuestionario es su rapidez en ser completado y calculado. Algunos estudios en pacientes con $\mathrm{AR}$, mostraron que el tiempo requerido para su cálculo es menor al necesario para calcular los índices compuestos DAS28 y CDAI ${ }^{22-25}$.

Concluimos que el cuestionario RAPID3 es un instrumento válido para ser utilizado en la consulta ambulatoria en pacientes con EsPax por la sencillez en la realización y cálculo, así como también por brindarnos conocimiento de tres aspectos importantes de la enfermedad como actividad de la enfermedad, capacidad funcional y calidad de vida.

\section{Bibliografía}

1. Garrett S, Jenkinson T, Kennedy LG, Whitelock H, Gaisford P, Calin A. A new approach to defining disease status in ankylosing spondylitis: the Bath Ankylosing Spondylitis Disease Activity Index. J Rheumatol 1994;21(12):2286-91.

2. Calin A, Garrett S, Whitelock H, Kennedy LG, O'Hea $\mathrm{J}$, Mallorie P, et al. A new approach to defining functional ability in ankylosing spondylitis: the development of the Bath Ankylosing Spondylitis Functional
Index. J Rheumatol 1994;21(12):2281-85.

3. Doward L, Spoorenberg A, Cook S, Whalley D, Helliwell P, Kay L, et al. Development of the ASQoL: a quality of life instrument specific to ankylosing spondylitis. Ann Rheum Dis 2003;62(1):20-6.

4. Pincus T, Askanase AD, Swearingen CJ. A Multi-dimensional Health Assessment Questionnaire (MDHAQ) and routine assessment of patient index data (RAPID3) scores are informative in patients with all rheumatic diseases. Rheum Dis Clin North Am 2009;35(4):819-27.

5. Pincus T, Yazici Y, Sokka T. Complexities in assessment of rheumatoid arthritis: absence of a single gold standard measure. Rheum Dis Clin North Am 2009;35(4):687-97.

6. Pincus T, Segurado O. An index based on only patient reported outcome (PRO) measures, without formal joint counts, routine assessment of patient index data (RAPID) classifies patients into 4 severity categories which are similar to Disease Activity Score (DAS28) and clinical Disease Activity Index (CDAI) categories in 4 adalimumab clinical trials [abstract]. Arthritis Rheum 2007;56 Suppl:S705-6.

7. Pincus T, Bergman MJ, Yazici Y, Hines P, Raghupathi $\mathrm{K}$, Maclean R. An index of only patient-reported outcome measures, routine assessment of patient index data 3 (RAPID3), in two abatacept clinical trials: similar results to disease activity score (DAS28) and other RAPID indices that include physician-reported measures. Rheumatology (Oxford) 2008; 47(3):345-49.

8. Castrejón I, Bergman MJ, Pincus T. MDHAQ/RAPID3 to recognize improvement over 2 months in usual care of patients with osteoarthritis, systemic lupus erythematosus, spondyloarthropathy, and gout, as well as rheumatoid arthritis. Journal of Clinical Rheumatology. 2013;19(4):169-74.

9. Maldonado Ficco H, Pérez Alamino R, Schneeberger EE, Maldonado Cocco JA, Citera G. Validación del cuestionario RAPID3 en una cohorte de pacientes con artritis reumatoidea temprana y establecida, y su correlación con otros índices de actividad. Rev Argent Reumatol 2011;22 (2):31-39.

10. Citera G, Maldonado Cocco J, Moroldo M, BurgosVargas R, Anaya J, López I, et al Validación de la versión en español de los cuestionarios de capacidad funcional (BASFI) y actividad de la enfermedad (BASDAI) en pacientes con Espondilitis Anquilosante en cuatro países latinoamericanos. Rev Argent Reumatol 1999;10(Supl 1):25 [abstract].

11. Marengo MF, Schneeberger EE, Citera G, Maldonado Cocco JA. Work status among patients with ankylosing spondylitis in Argentina. J Clin Rheumatol 
2008;14(5):273-7

12. Heuft-Dorenbosch L, Spoorenberg A, van Tubergen A, Landewé $\mathrm{R}$, van der Tempel $\mathrm{H}$, Mielants $\mathrm{H}$, et al. Assessment of enthesitis in ankylosing spondylitis. Ann Rheum Dis 2003; 62(2):131-36.

13. Lukas C, Landewé R, Sieper J, Dougados M, Davis J, Braun J, et al. Assessment of SpondyloArthritis international Society et al. Development of an ASAS-endorsed disease activity score (ASDAS) in patients with ankylosing spondylitis. Ann Rheum Dis 2009;68(1):1824.

14. Machado P, Landewé R, Lie E, Kvien TK, Braun J, Baker D, et al. Assessment of SpondyloArthritis international Society et al. Ankylosing spondylitis disease activity score (ASDAS): defining cut-off values for disease activity states and improvement scores. Ann Rheum Dis 2011;70(1):47-53.

15. Buschiazzo E, Sommerfleck F, Schneeberger EE, Arturi P, Maldonado Cocco JA, Citera G. Validación del índice ASDAS en una cohorte de pacientes con Espondilitis Anquilosante. Rev Arg Reumatol 2010;21:28.

16. Sommerfleck FA, Schneeberger EE, Buschiazzo EE, Maldonado Cocco JA, Citera G. A simplified version of ankylosing spondylitis disease activity score (ASDAS) in patients with ankylosing spondylitis. Clin Rheumatol 2012;31(11):1599-603.

17. Salaffi F, Ciapetti A, Carotti M, Gasparini S, Citera G, Gutiérrez M. Construct validity and responsiveness of the simplified version of ankylosing spondylitis disease activity score (SASDAS) for the evaluation of disease activity in axial spondyloarthritis. Health Qual Life Outcomes 2014;12:129.

18. MacKay K, Mack C, Brophy S, Calin A. The Bath ankylosing spondylitis radiology index (BASRI): A new, validated approach to disease assessment. Arthritis Rheum 1998;12(41): 2263-70.

19. Danve A, Reddy A, Vakil-Gilani K, Garg N, Dinno A, Deodhar A. Routine Assessment of Patient Index Data 3 score (RAPID3) correlates well with Bath Ankylosing Spondylitis Disease Activity index (BASDAI) in the assessment of disease activity and monitoring progression of axial spondyloarthritis. Clin Rheumatol 2015;34(1):117-24.

20. Cinar M, Yilmaz S, Cinar FI, Koca SS, Erdem H, Pay $\mathrm{S}$, et al. A patient-reported outcome measures-based composite index (RAPID3) for the assessment of disease activity in ankylosing spondylitis. Rheumatol Int 2015;35(9):1575-80.

21. Park SH, Choe JY, Kim SK, Lee H, Castrejón I, Pincus T. Routine Assessment of Patient Index Data (RAPID3) and Bath Ankylosing Spondylitis Disease
Activity Index (BASDAI) Scores Yield Similar Information in 85 Korean Patients With Ankylosing Spondylitis Seen in Usual Clinical Care. J Clin Rheumatol 2015;21(6):300-4.

22. Yokogawa N, Kaneko T, Nagai Y, Nunokawa T, Sawaki T, Shiroto K, et al. Validation of RAPID3 using a Japanese version of Multidimensional Health Assessment Questionnaire with Japanese rheumatoid arthritis patients: characteristics of RAPID3 compared to DAS28 and CDAI. Mod Rheumatol 2015;25(2):264-9.

23. Singh H, Gupta V, Ray S, Kumar H, Talapatra P, Kaur $\mathrm{M}$, et al. Evaluation of disease activity in rheumatoid arthritis by Routine Assessment of Patient Index Data 3 (RAPID3) and its correlation to Disease Activity Score 28 (DAS28) and Clinical Disease Activity Index (CDAI): an Indian experience. Clin Rheumatol 2012;31(12):1663-9.

24. Kim SK, Park SH, Bae J, Son JT, Choe JY. Performance of Routine Assessment of Patient Index Data 3 (RAPID3) for assessment of rheumatoid arthritis in clinical practice: differential agreement of RAPID3 according to disease activity categories. Rheumatol Int 2014;34(9): 1311-8.

25. Yazici Y, Bergman M, Pincus T. Time to score quantitative rheumatoid arthritis measures: 28-joint count, disease activity score, health assessment questionnaire (HAQ), multidimensional HAQ (MDHAQ), and routine assessment of patient index data (RAPID) scores. J Rheumatol 2008;35(4):603-9. 